\title{
The factor structure and use of the Demoralization Scale (DS-IT) \\ in Italian cancer patients
}

Luigi Grassi, ${ }^{1,2}$ Anna Costantini, ${ }^{3}$ David Kissane, ${ }^{4}$ Serena Brunetti, ${ }^{3}$ Rosangela Caruso,, 2 Giulia Piazza, ${ }^{1}$ Paolo Marchetti, ${ }^{5}$ Silvana Sabato, ${ }^{1}$ Maria Giulia Nanni ${ }^{1,2}$

${ }^{1}$ Institute of Psychiatry, Department of Biomedical and Specialty Surgical Sciences, University of Ferrara, Ferrara, Italy and ${ }^{2}$ University Hospital Psychiatry Unit, Integrated Department of Mental Health and Addictive Behaviors, Health Authorities and University S. Anna Hospital, Ferrara, Italy luigi.grassi@unife.it; rosangela.caruso@unife.it; mariagiulia.nanni@unife.it; giulia.piazza@unife.it; silvana.sabato@unife.it

${ }^{3}$ Psychoncology Unit, Sant'Andrea Hospital Sapienza, University of Rome, Rome Italy anna.costantini@ospedalesantandrea.it; serenabrunetti@libero.it

${ }^{4}$ Department of Psychiatry, Monash University and Szalmuk Family Research Unit at Cabrini Health, Victoria, Australia david.kissane@ monash.edu

${ }^{5}$ Medical Oncology, La Sapienza University of Rome and IDI - IRCCS, Rome, Italy paolo.marchetti@ospedalesantandrea.it

\section{Correspondence address:}

Luigi Grassi, M.D.

Clinica Psichiatrica

Università di Ferrara

Corso Giovecca 203

44100 Ferrara, Italy

Tel +39 0532 236809; Fax +390532 212240; e-mail luigi.grassi@unife.it

This is the author manuscript accepted for publication and has undergone full peer review but has not been through the copyediting, typesetting, pagination and proofreading process, which may lead to differences between this version and the Version of Record. Please cite this article as doi: $10.1002 /$ pon. 4413

This article is protected by copyright. All rights reserved. 


\section{Abstract}

Background: Demoralization is a commonly observed syndrome in cancer patients, deserving to be carefully assessed in cross-cultural contexts.

Aims: To examine the factor structure, concurrent and divergent validity of the Italian version of the Demoralization Scale (DS-IT) in cancer patients.

Methods: The sample included 194 Italian cancer outpatients who were assessed by using the DSIT and the Diagnostic Criteria of Psychosomatic Research-demoralization module (DCPR/D) to examine demoralization. The Patient Health Questionnaire-9 (PHQ-9) to explore depression, and the Mini-Mental Adjustment-to-Cancer-Hopelessness scale (Mini-MAC-HH) to explore maladaptive coping were also administered.

Results: Four factors were extracted by exploratory factor analysis on the DS-IT (Disheartenment, $\alpha=0.87$; Sense of Failure, $\alpha=0.77$; Dysphoria, $\alpha=0.73$; Loss of Meaning/Purpose, $\alpha=0.72$; Total $\alpha=0.91$ ), accounting for $57.1 \%$ of the variance. The DS-IT factors shared between $17 \%$ and $36 \%$ of the variance. Patients reporting a diagnosis of demoralization on the DCPR/D (23.7\%) had higher scores on DS-IT Loss of Meaning/Purpose, Sense of Failure, Dysphoria and DS-IT Total. About half of those who were highly demoralized were not depressed and among those who had moderate or moderately severe demoralization, about $80 \%$ were not depressed on the PHQ-9. The DS-IT was significantly associated with PHQ-9 and Mini-MC-HH.

Conclusions: The study presents further evidence that demoralization is a significant clinical condition and that the DS-IT demonstrates satisfactory levels of validity and reliability to support its use in patients in the ambulatory cancer setting.

Key words: demoralization, depression, cancer, oncology, psychiatry, psycho-oncology 


\section{Introduction}

Studies over the last thirty years have indicated that $30-40 \%$ of cancer patients meet the criteria for a psychiatric diagnosis, especially in the area of depressive spectrum disorders, with negative consequences for the patients and their families.[1] More recently, research has focused attention on demoralization as a specific psychosocial syndrome not detectable by using classical psychiatric nosology.[2] It defines a state of existential distress denoting a persistent failure of coping with stress occurring in patients with severe physical illness or mental conditions, specifically ones that threaten life or the integrity of being.[3] In medical settings, including oncology and palliative care, demoralization has been described as a combination of distress and subjective incompetence; loss of meaning and purpose in life; cognitive attitudes of pessimism, hopelessness/helplessness, sense of being trapped, personal failure; with associated features of social alienation or isolation and lack of support.[4,5,6,7,8]

Studies have shown that demoralization can be diagnosed in $15 \%$ to $30 \%$ of patients in oncology and palliative care settings, according to the type of instrument.[9,10] Demoralization can also be clinically separated from depressive disorders [11] and has a remarkable role in negatively influencing a patients' quality of life, coping styles, and dignity.[12,13,14] Recent data show that it is also associated with suicidal ideation to a greater extent than clinical depression, [15] and thus needs to be carefully examined, correctly measured and treated.[16]

On this background, the Demoralization scale (DS) has been developed, originally validated among 100 advanced cancer patients, [17] and shown to capture the core dimensions of demoralization as proposed by Clarke and Kissane, [8] namely loss of meaning, dysphoria, disheartenment, helplessness, and sense of failure. Within oncology and palliative care settings, the DS has been translated and applied in several countries, such as Germany,[18] Ireland,[19] Taiwan,[20] Italy,[21] and Spain,[22] with data confirming its construct and convergent validity with respect to other psychometric instruments, although the factor structure of the DS was different with respect to the original.[18,20]

The aims of the present study were to extend previous preliminary data [21] on the validity and application of the Italian version of the DS (DS-IT) not only in palliative care settings, but also in cancer patients seen in ambulatory settings, as this mental state contributes to substantial 
emotional and cognitive states of distress. We examined the psychometric properties of the scale, including the factor structure and replicability, internal consistency, and concurrent and divergent validity through associations with and group differences between another interview-based measure of demoralization, as well as hopelessness and depression.

\section{Methods}

The study was carried out at the Department of Clinical Oncology of the Sant'Andrea University Hospital in Rome, and the Sant'Anna University Hospital in Ferrara, Italy. Inclusion criteria were: (i) age $\geq 18$ and $\leq 70$; (ii) cancer diagnosis (all stages) at least after one year since diagnosis; (iii) Karnofsky Performance Status Scale (KPS) $\geq 80$ (to analyse demoralization specifically in patients with good performance status); and (iv) absence of cognitive disorders as assessed by clinical evaluation. A convenience sample of patients, after providing written informed consent, was recruited at cancer outpatient clinics and day-hospitals, and asked by a research assistant to complete questionnaires and participate in a clinical semi-structured interview. Both the questionnaires and the interview took about 45 minutes to be completed. The study was approved by the Ethical Review Committee for Human Research of the participating institutions.

\section{Instruments}

The DS-IT [17] was used in its 24 item-format, each item rated on five-point Likert scale ("never"=0; "all the time"=4) over the past two weeks. Five subscales were derived in the original study, specifically Loss of Meaning/Purpose $(\alpha=0.87)$; Dysphoria $(\alpha=0.85)$; Disheartenment ( $\alpha=0.89)$; Helplessness $(\alpha=0.84)$, and Sense of failure $(\alpha=0.71)$. A total score $(\alpha=0.94$; range $0-96)$ is obtained by summing the single sub-scales scores. As reported elsewhere [21], the DS was translated forward and backward into the Italian language, with the support of a native English speaker.

The Patient Health Questionnaire [23] 9-items (PHQ-9), in its Italian version [24] was used to assess depression. The PHQ-9 is based on the Diagnostic and Statistical Manual of Mental Disorders-IV diagnostic criteria (DSM) for major depressive disorder. Each item is rated on a fourpoint Likert scale $(0=$ not at all; $3=$ nearly every day) over the past two weeks. The psychometric 
properties of the scale has been repeatedly shown,[25] with a cut-off point of $\geq 10$ recommended for the screening of depression [26]. In this study the Cronbach's $\alpha$ of the PHQ-9 was acceptable-good $(\alpha=.80)$.

The Mini-Mental Adjustment to Cancer Hopelessness-Helplessness (HH) subscale (Mini$\mathrm{MAC} / \mathrm{HH}$ ) was used in its 8 item-format, extracted from the Mini-MAC scale, [27] in its Italian version. [28] Each item measures, on a 4-point Likert scale (range 8-32), the tendency to adopt a pessimistic and hopeless coping style. The Mini-MAC/HH has been validated in Italian cancer patients, showing good psychometric properties $(\alpha=.92)$. $[29,30]$

Each patient was also administered a semi-structured interview according to the Diagnostic Criteria for Psychosomatic Research-Demoralization module (DCPR/D).[6,31] A DCPR/D diagnosis is made if the following criteria are met: (A) feeling unable to cope with pressing problems and aware of having failed to meet his/her own expectations or those of others; (B) feeling helpless, hopeless or wanting to give up; (D) this mental state is prolonged and generalized (duration $\geq$ one month). The DCPR/D has been validated in a number of studies in medically ill patients [32,33], including cancer patients.[12,13]

The patients socio-demographic (age, education, marital status, and occupation) and medical information (site and stage of cancer, treatment, time since diagnosis, and KPS score) were gathered from the patients' clinical charts.

\section{Statistical Analysis}

To investigate the underlying DS-IT structure, we used an exploratory factor analysis using the principal-factor method with orthogonal varimax rotation. This approach is consistent with the method used in DS validation studies [17-22] carried out in both advanced and non-advanced cancer patients. Two tests confirmed suitability for factor analysis - a Kaiser-Meyer-Olkin > 0.6 showed adequate sampling,[34] and a significant Bartlett $\alpha$ value confirmed sphericity.[35] We used 3 criteria to guide how many factors to keep: a Horn parallel analysis,[36] inspection of the Cattell scree plot and eigenvalues $>1$.[37] Internal consistency was estimated by calculating Cronbach's coefficient alpha for each scale. Concurrent validity was analyzed using Spearman's rho 
correlation test. We examined the prevalence of low, moderate and high demoralization by determining the DS-IT cut-off scores according to Mullane et al.[19] (low scorers, <mean-1SD; middle scorers, mean-1SD to mean+1SD; and high scorers, >mean+1SD) and Robinson et al.,[38] (low scorers, 0-25th percentile; middle scorers, 25th-75th percentile; and high scorers, $>75$ th percentile), in order to have a comparable description with what has been reported in other studies. However, since the cut-off for moderate demoralization generally leads to a very high percentage of moderately demoralized patients, with questionable clinical relevance, discriminant validity between demoralization and depression was examined by determining the DS cut-off scores with reference to an extreme groups design. [34] More specifically, the cut-off for demoralization was dropped off in several classes (no/low demoralization, <mean-1SD; moderate demoralization, 25th75th percentile; moderately severe demoralization, 75 th percentile to mean+1SD; and severe demoralization, >mean+1SD). T-test, chi-square and ANOVA were employed to determine the differences between groups when comparing demoralization with psychosocial, clinical and sociodemographic variables. Spearman's rho correlation test was used to analyse association between variables. The Statistical Package for Social Sciences (SPSS) version 20 was used for analysis, with the level of statistical significance set at $p<0.05$.

\section{Results}

A total of 210 patients meeting the inclusion criteria were approached over the study period. Of these, 13 declined participation for several reasons (5 no interest; 7 lack of time; 1 diseaserelated problems) and 3 had missing measures $(<5 \%)$ not allowing evaluation. The final sample was composed of 194 subjects, mostly female (67\%), mean aged 55 years, 50\% with early stage and $50 \%$ metastatic cancer, and an average of 2.2 years since diagnosis (see online supplement Table 1).

\section{Factor structure and internal consistency of the DS}

The Kaiser-Meyer-Olkin measure of sample adequacy was 0.91 , indicating that the factor analysis was appropriate. Principal component analysis (Varimax rotation with Kaiser normalization) identified 4 factors, all of which had eigenvalues $\geq 1.0$, explaining $57.1 \%$ of the variance (Table 1). The first factor, Disheartenment, consisted of 8 items, inclusive of five items 
(items 18,21,22,23,24) of the original DS-Disheartenment scale, plus three items (items 5,8,9), belonging to the original DS-Helplessness subscale. The second factor, Sense of Failure, comprised the same items of the original DS-Sense of Failure scale (items 1,2,17,19), plus one item that originally loaded on the DS-Disheartenment subscale (item 6). The third factor, Dysphoria, consisted of the same five items as the original DS-Dysphoria scale (items 10,11,13,15,16). The fourth factor, Loss of Meaning/Purpose, consisted of the same items of the original scale (items 2,3, $4,14,20$ ), plus one item (item 7) that originally loaded on the DS-Helplessness subscale. The latter subscale was not replicated as a single factor in our study. Cronbach's $\alpha$-coefficients indicated good (DS-IT Total $\alpha=0.91$, Disheartenment $\alpha=0.87$ ) or acceptable levels of internal consistency (Sense of Failure, $\alpha=0.77$, Dysphoria $\alpha=0.73$, Loss of Meaning/Purpose, $\alpha=0.72$ ).

Subscale Inter-correlations and prevalence of demoralization

High inter-correlations were found among the single DS-IT dimensions (Table 2). The Disheartenment subscale shared 36\% of the variance with Loss of Meaning/Purpose and Dysphoria, and $17 \%$ with Sense of Failure. The Loss of Meaning/Purpose subscale shared $20 \%$ of the variance with Dysphoria and $18 \%$ with Sense of Failure. The Dysphoria subscale shared $17 \%$ of the variance with Sense of Failure. High statistically significant correlations were also found between the individual DS-IT subscales and DS-IT Total.

The DS-IT Total had a mean score of 23.55 \pm 14.1 . Following the methodology of Mullane et al.,[19] 27 patients (13.9\%) were classified as having no/low demoralization, 134 patients (69.1\%) moderate demoralization, and 33 patients (17\%) high demoralization. Following Robinson et al.,[35] 43 patients (22.2\%) were classified as having low demoralization, 104 patients (53.6\%) moderate demoralization and 47 patients $(24.2 \%)$ high demoralization (see online supplement Table 2).

\section{Concurrent Validity}

The patients who were positive "cases" for demoralization using the DCPR/D criteria $(\mathrm{n}=46 / 194,23.7 \%)$ showed significantly higher scores on the DS-IT-Loss of Meaning/Purpose $(\mathrm{F}=5.8, \mathrm{df} 1, \mathrm{p}=0.01)$, DS-IT-Failure $(\mathrm{F}=4.5, \mathrm{df} 1, \mathrm{p}=0.03)$, DS-IT-Dysphoria $(\mathrm{F}=4.1, \mathrm{df} 1, \mathrm{p}=0.04)$, 
and DS-IT-Total ( $\mathrm{F}=4.75$, $\mathrm{df} 1, \mathrm{p}=0.03$ ) (see online supplement Table 3), while on DS-ITDisheartenment, the differences did not reach a level of statistical significance $(\mathrm{F}=2.7, \mathrm{p}=0.1)$. Also significant associations were found between all dimensions of DS-IT and both PHQ-9 (r from 0.41 to $0.65, \mathrm{p}=0.001$ ) and Mini-MAC/HH scores ( $\mathrm{r}$ from 0.33 to $0.54, \mathrm{p}=0.001$ ) (Table 2). Highly demoralized patients showed, with respect to those with low demoralization, higher scores on PHQ9 (Mullane's method: $\mathrm{F}=41.8$; df2, $\mathrm{p}=0.001$; Robinson's method: $\mathrm{F}=37.4$, df2, $\mathrm{p}=0.001$ ) and MiniMAC/HH (Mullane's method: F=20.56, df2, p=0.001; Robinson's method: $F=25.85$,df2, p=0.001).

\section{Divergent Validity}

As planned (see statistical analysis), divergent validity between the constructs of demoralization and depression was explored by examining cross-tabulation frequencies of PHQ-9 cases of depression with patients having no/very low $(n=43,22.2 \%)$, moderate $(n=104,53.6 \%)$, moderately severe $(n=17,7.2 \%)$ and severe demoralization $(n=33,17 \%)$. According to the PHQ-9 cut-off ( $\geq 10), 20.6 \%$ were classified as "cases" of depression. Sixteen patients who were severely demoralized were non-cases on the PHQ-9 (16/43, 48.5\%), while among those who were moderately severe demoralized or moderately demoralized, $11(11 / 17,78.5 \%)$ and $85(85 / 104$, $82 \%)$ respectively, did not show any symptom of depression, $(\chi 2,28.8$, df 3 , p=0.0001) (Table 3 ). Association of demoralization with socio-demographic and medical variables.

Analyzing the correlation of the DS-IT with socio-demographic and clinical variables, no significant correlations were found with age (besides a marginally significant association between age and DSI-IT-Loss of Meaning/Purpose, rho=.17, $\mathrm{p}<0.05$ ). No association was found on the mean scores on any DS-IT and marital status, education, and sex, besides a marginally significantly higher score on DS-IT-Dysphoria in females than males $(\mathrm{F}=4.03 ; \mathrm{p}=0.04)$. No association was found with KPS score, site and stage of cancer. Time since diagnosis was significantly associated with DS-IT-Disheartenment (rho=.19, p<0.05), Dysphoria (rho=.22, p<0.01), Failure (rho=.17, $\mathrm{p}<0.05)$ and Total $(\mathrm{rho}=.22, \mathrm{p}<0.01)$ (see online supplement Table 2).

\section{Discussion}

Demoralization has become an important psychosocial construct to be taken into account in cancer patients $[9,10,16]$. This study aimed to cross-culturally investigate the factor structure and 
the psychometric properties of the DS-IT [21] in a sample of 194 patients with both early stage and advanced cancer and good performance status. This validation of the DS-IT has not only included patients in the palliative/advanced cancer setting, but additionally included cancer patients seen in ambulatory settings, as this mental state contributes to substantial emotional and cognitive states of distress.

The results of the exploratory factor analysis demonstrated a four-dimensional factor structure (i.e. Disheartenment, Sense of Failure, Dysphoria, and Loss of Meaning/Purpose), which explained $57.1 \%$ of the variance. The factor solution in this analysis revealed some different item clustering to the English scale validated by Kissane et al.[17] and Mullane et al.[19] but were in precise agreement with the German version,[18] which also showed four factors with the same content as we found. In terms of items loading, Dysphoria and Loss of Meaning/Purpose consisted of exactly the same factors of the original version; Sense of Failure also comprised the same items of the original DS plus one item (Item 6 "I am in good spirits", reversed item) which has consistent face validity with the sense of failure subscale. Two items of the original Helplessness dimension (Item 5, "I no longer feel emotionally in control"; Item 9, “I feel hopeless") loaded on the factor Disheartenment, while item 7 ("No one can help me") loaded on the factor Loss of Meaning/Purpose. While these items are all spread across the dimensional nature of demoralization, cultural influences may result in the first two items loading on disheartenment (at the milder end of the demoralized construct) and the third item at the more severe end of the spectrum (Loss of Meaning/Purpose). Cronbach's $\alpha$-coefficients between 0.79 and 0.90 for the single factors and 0.92 for the total score also indicated acceptable to excellent levels of internal consistency for the scale. There were statistically significant correlations between some factors, especially Disheartenment and both Lack of Meaning/Purpose and Dysphoria, with 36\% of the variance explained.

Regarding the mean score of the DS-IT, comparable data were found with what reported in a sample of Irish palliative care patients (19.94 \pm 14.62$)$, although lower than that reported in German $(29.80 \pm 10.41)$ and Australian $(30.82 \pm 17.73)$ studies. The last result could be explained by the fact that our sample was in a good performance cohort with respect to the mentioned studies. When examining the prevalence of demoralization, we used different methods, as recommended by Mullane et al.[19] (cut-off score based on the DS-IT-Total mean \pm 1 SD) and Robinson et al.[39] (0- 
$25^{\text {th }}, 25^{\text {th }}-75^{\text {th }},>75^{\text {th }}$ percentile). The percentage of patients with high $(17 \%)$ and moderate levels $(69.1 \%)$ of demoralization was similar to that reported in palliative care settings both in Germany $(15.7 \%$ and $73.1 \%)$ and Ireland (14\% and 68\%). However, one problem that arises when using cutoff scores is that a high percentage of patients are found to be moderately demoralized, with questionable relevance in terms of clinical practice. Thus, when splitting the population according to a mixed method (no/very low, <mean-1SD; moderate, scores 25 th-75th percentile, moderately severe, scores 75 th percentile -mean+1SD; and severe, $>$ mean+1SD), we identified only a small subgroup of patients ( $7.2 \%)$ that were in an intermediate area (moderately severe demoralization). More studies are necessary to better qualify the continuum of demoralization in clinical care. However, our findings are important since they confirm that demoralization is a common syndrome that can be observed in all trajectories of cancer, including patients with local or loco-regional disease and with a good performance level.

Regarding concurrent validity, interesting results were found when exploring the relationship between DS-IT and the demoralization construct, as measured by the DCPR/D interview. The percentage of patients that met the criteria for a DCPR/D diagnosis of demoralization was similar to that reported in other studies using the DCPR/D in non-advanced cancer patients [12,13]. With respect to the DS-IT, higher scores on Meaninglessness, Dysphoria, Failure, and DS-IT-Total were found in DCPR/D demoralized patients. More specifically, the DCPR/D criterion A (feeling that one failed to meet his/her own expectations and those of others) could be measured by the DS-IT-Failure dimension. Also the content of DCPR/D criterion B (feeling helpless/hopeless or wanting to give up) is at least in part reproduced by the DS-IT-Loss of Meaning/Purpose factor. More studies are however necessary, given other differences existing between the two instruments that should be taken into account. Disheartenment represents a mild lowering of morale, which may not be pathological in itself, and thus it makes sense that this feature is not included within the DCPR/D diagnostic criteria. A dimensional measure such as the DS-IT needs to capture the mild end of the construct without implying that morbidity exists, while a categorical set of criteria, like the DCPR/D, should only include items that are pathological. In this manner, this variation between the two systems is acceptable. Further, the DCPR/D criterion C (the condition is prolonged and generalized for at least one month in duration) is different from what the DS-IT investigates, that is the last two weeks, the latter time frame being selected to correspond 
with DSM constructs. In practice, any such time frame is arbitrary, needing to be sufficiently long to differentiate from transient mental states, yet not so long as to extend suffering in the absence of treatment. Regarding hopelessness/helplessness, more research is necessary, since this factor present in the original DS was not shown in the present study. However, interesting association were found between the Mini-MAC/HH and the other DS-IT factors. Also, patients with high levels of demoralization showed high scores on Mini-MAC/HH scale, irrespective of the rating method for demoralization (i.e., Mullane's or Robinson's). This indicates that dysfunctional coping mechanisms are part of the demoralization construct among cancer patients, adding new information about the negative consequences of demoralization [13-15].

As regards divergent validity, we examined the differences between patients showing clinical depression on the PHQ-9 (20.6\% in our sample) and demoralization. Just as both anxiety and depression can exist co-morbidly in some patients, we would expect some level of co-morbidity between demoralization and depression. In fact, significant correlations were found between the DS-IT and the PHQ-9 (rho from 0.41 to 0.65 ); however this overlap is expected and does not reduce the clinical value of the demoralization concept since almost half of patients with severe demoralization and more than three quarter of those who had moderate or moderately severe demoralization were not clinically depressed at the PHQ-9 (cut-off $\geq 10$ ). These findings confirm previous studies carried out both in the medically ill [33] and cancer patients [17-20] showing that demoralization is a different construct to depression, although some overlap is more evident when the severity of depression increases.

An interesting result is also that, whereas demoralization did not appear to be related to site, stage and treatment in our sample, it was associated with time since diagnosis, indicating that with time, the resources and capacities to cope with the stress of cancer may decrease and patients can be more prone to develop a demoralization syndrome. This should alert physicians to assess morale throughout the trajectory of the disease.

\section{Study Limitations}

There are limitations to our study. Replication is needed in a larger population with more representative cancer sites, different stages of illness, and lower KPS status, thus examining patients 
who might have higher demoralization because of their greater physical symptom burden. Further research could explore the association between demoralization and other psychosocial dimensions (e.g. personality traits, existential and spiritual variables),[39] as well as the outcome of demoralization in terms of quality of life.[40] Last, a new version II of the DS (DS-II) has recently been validated in Australia [38,41] after our study was conducted. Although our findings add valuable information about demoralization in an Italian sample, replication of the DS-II is Italy also possible.

\section{Clinical implications}

The strength of this study is its replication of the importance of demoralization in both advanced and non-advanced cancer patients, allowing the use of the DS-IT in many cancer settings, and not only palliative care. Also, this is the first study examining a possible correlation between the DS-IT and another measure of demoralization, the DCPR/D, that has been already applied in cancer settings.[12,13] Future research can use the DCPR/D as a semi-structured interview to identify the threshold of clinical relevance when demoralization is measured by the DS-IT.

ACKNOWLEDGEMENTS. The authors wish to thank all the patients participating in the study. The research group in Ferrara (LG, RC, SS, GP, MGN) would like to express their gratitude to Unitalsi Triveneta and the Italian Medical Board/Association - Section of Ferrara for their unrestricted research grant and support in the memory of Francesco Tomasi, M.D., and the Associazione per Supporto Psico-Oncologico (ASPO) for their unrestricted clinical research grant for the improvement of psychosocial care in oncology.

This article is protected by copyright. All rights reserved. 


\section{References}

1 Caruso R, Nanni MG, Riba M, Sabato S, Mitchell AJ, Croce E, Grassi L.: Depressive spectrum disorders in cancer: prevalence, risk factors and screening for depression: a critical review. Acta Oncol 2017;56(2):146-155

2 Tecuta L, Tomba E, Grandi S, Fava GA.: Demoralization: a systematic review on its clinical characterization. Psychol Med. 2015;45(4):673-91.

3 de Figueiredo JM.: Demoralization and Psychotherapy: A Tribute to Jerome D. Frank, MD, PhD (1909-2005).Psychother Psychosom. 2007;76(3):129-33.

4 de Figueiredo JM, Frank JD.: Subjective incompetence, the clinical hallmark of demoralization. Compr Psychiatry.1982; 23(4):353-63

5 Fava GA, Freyberger HJ, Bech P, Christodoulou G, Sensky T, Theorell T, Wise TN.: Diagnostic criteria for use in psychosomatic research. Psychother Psychosom. 1995;63(1):1-8.

6 Fava GA, Mangelli L, Ruini C.: Assessment of psychological distress in the setting of medical disease. Psychother Psychosom. 2001;70(4):171-5.

7 Clarke DM, Kissane DW. Demoralization: its phenomenology and importance. Australian and NewZealand Journal of Psychiatry, 2002; 36: 733-742.

8 Kissane DW, Clarke DM, Street AF.: Demoralization syndrome--a relevant psychiatric diagnosis for palliative care. J Palliat Care. 2001;17:12-21.

9 Robinson S, Kissane DW, Brooker J, Burney S.: A systematic review of the demoralization syndrome in individuals with progressive disease and cancer: a decade of research. J Pain Symptom Manage. 2015;49:595-610

10 Tang PL, Wang HH, Chou FH.: A Systematic Review and Meta-Analysis of Demoralization and Depression in Patients With Cancer. Psychosomatics. 2015;56(6):634-43.

11 de Figueiredo JM. Depression and demoralization: phenomenologic differences and research perspectives. Compr Psychiatry 1993;34(5):308-11.

12 Grassi L., Sabato S., Rossi E., Biancosino B., Marmai L: The use of the Diagnostic Criteria for Psychosomatic Research (DCPR) in oncology. Psychother Psychosom 2005;74:100-107

13 Grassi L., Rossi E., Sabato S., Cruciani G., Zambelli M.: Diagnostic Criteria for Psychosomatic Research (DCPR) and psychosocial variables in breast cancer patients. Psychosomatics 2004; 45:483-491

14 Vehling S, Mehnert A Symptom burden, loss of dignity, and demoralization in patients with cancer: a mediation model. Psychooncology. 2014;23(3):283-90. 
15 Fang CK, Chang MC, Chen PJ, Lin CC, Chen GS, Lin J, Hsieh RK, Chang YF, Chen HW, Wu CL, Lin KC, Chiu YJ,Li YC.: A correlational study of suicidal ideation with psychological distress, depression, and demoralization in patients with cancer. Support Care Cancer. 2014;22(12):3165-74.

16 Grassi L., Nanni M.G.: Demoralization Syndrome: New Insights in Psychosocial Cancer Care. Cancer, 2016; 122(14):2130-2133

17 Kissane DW, Wein S, Love A, Lee XQ, Kee PL, Clarke DM.: The Demoralization Scale: a report of its development and preliminary validation. J Palliat Care. 2004;20:269-276.

18 Mehnert A, Vehling S, Höcker A, Lehmann C, Koch U.: Demoralization and depression in patients with advanced cancer: validation of the German version of the demoralization scale. J Pain Symptom Manage. 2011;42(5):768-76

19 Mullane M, Dooley B, Tiernan E, Bates U.: Validation of the Demoralization Scale in an Irish advanced cancer sample. Palliat Support Care. 2009;7(3):323-30.

20 Lee CY, Fang CK, Yang YC, Liu CL, Leu YS, Wang TE, Chang YF, Hsieh RK, Chen YJ, Tsai LY, Liu SI, Chen HW.: Demoralization syndrome among cancer outpatients in Taiwan. Support Care Cancer. 2012;20(10):2259-67.

21 Costantini A, Picardi A, Brunetti S, Trabucchi G, Bersani FS, Minichino A, Marchetti P. [Italian version of Demoralization Scale: a validation study]. Riv Psichiatr. 2013;48(3):234-9

22 Rudilla D, Galiana L, Oliver A, Barreto P.: Demoralization Scale in Spanish-Speaking Palliative Care Patients. J Pain Symptom Manage. 2016;51(4):769-775.

23 Spitzer RL1, Kroenke K, Williams JB. Validation and utility of a self-report version of PRIMEMD: the PHQ primary care study. Primary Care Evaluation of Mental Disorders. Patient Health Questionnaire. JAMA. 1999;282(18):1737-44.

24 Rizzo R, Piccinelli M, Mazzi MA, Bellantuono C, Tansella M.: The Personal Health Questionnaire: a new screening instrument for detection of ICD-10 depressive disorders in primary care. Psychol Med. 2000;30(4):831-40.

25 Thombs BD, Benedetti A, Kloda LA, Levis B, Nicolau I, Cuijpers P, Gilbody S, Ioannidis JP, McMillan D, Patten SB, Shrier I, Steele RJ, Ziegelstein RC. : The diagnostic accuracy of the Patient Health Questionnaire-2 (PHQ-2), Patient Health Questionnaire-8 (PHQ-8), and Patient Health Questionnaire-9 (PHQ-9) for detecting major depression: protocol for a systematic review and individual patient data meta-analyses. Syst Rev. 2014;3:124. doi: 10.1186/2046-4053-3-124.

26 Kroenke K, Spitzer RL, Williams JBW. The PHQ-9, validity of a Brief Depression Severity Measure. J Gen Int Med, 16:606-13, 2001.

27 Watson, M., Law, M., dos Santos, M., Greer, S., Baruch, J.\& Bliss, J. The Mini-MAC: further development of the mental adjustment to cancer scale. Journal of Psychosocial Oncology, 1994; 12: 
$33-44$.

28 Grassi L., Buda P, Cavana L, Annunziata MA, Torta R, Varetto A.: Styles of coping with cancer: the Italian version of the Mini-Mental Adjustment to Cancer (Mini-MAC) scale. Psychooncology. 2005;14:115-124.

29 Grassi L., Travado L., Gil F., Sabato S., Rossi E., Tomamichel M., Marmai L., Biancosino B., Nanni M.G. and the SEPOS Group: Hopelessness and Related Variables Among Cancer Patients in the Southern European Psycho-Oncology Study (SEPOS). Psychosomatics 2010; 51:201-207

30 Grassi L, Berardi MA, Ruffilli F, Meggiolaro E, Andritsch E, Sirgo A, Caruso R, Juan Linares E, Bellé M, Massarenti S, Nanni MG; IOR-IRST Psycho-Oncology and UniFE Psychiatry CoAuthors: Role of psychosocial variables on chemotherapy-induced nausea and vomiting and healthrelated quality of life among cancer patients: a European study. Psychother Psychosom. 2015;84(6):339-47

31 Fava GA, Cosci F, Sonino N.: Current Psychosomatic Practice. Psychother Psychosom. 2017;86(1):13-30.

32 Mangelli L, Fava GA, Grandi S, Grassi L, Ottolini F, Porcelli P, Rafanelli C, Rigatelli M, Sonino N. Assessing demoralization and depression in the setting of medical disease. J Clin Psychiatry, 66(3):391-4, 2005.

33 Grassi L, Mangelli L, Fava GA, Grandi S, Ottolini F, Porcelli P, Rafanelli C, Rigatelli M, Sonino N.: Psychosomatic characterization of adjustment disorders in the medical setting: some suggestions for DSM-V. J Affect Disord. 2007;101(1-3):251-254.

34 Kaiser HF. A second generation little jiffy. Psychometrika. 1970;35: 401-415.

35 Bartlet M. A note on multiplying factors for various chi-squared approximations. J R Stat Soc B. 1954;16:296-298.

36 Horn JL. A rationale and test for the number of factors in factor analysis. Psychometrika. 1965;30:179-185.

37 Preacher KJ. Extreme groups designs. In: Cautini RL, Lilienfeld SO, eds. The Encyclopedia of Clinical Psychology. Vol. 2. Hoboken, NJ: John Wiley \& Sons, Inc; 2015:1189-11924

38 Robinson S, Kissane DW, Brooker J, Hempton C, Michael N, Fischer J, et al.: Refinement and revalidation of the demoralization scale: The DS-II-external validity. Cancer. 2016;122(14):22602267

39 Vehling S, Lehmann C, Oechsle K, Bokemeyer C, Krüll A, Koch U, Mehnert A. Global meaning and meaning-related life attitudes: exploring their role in predicting depression, anxiety, and demoralization in cancer patients. Support Care Cancer, 2011; 19(4):513-520.

40 Grassi L, Biancosino B, Marmai L, Rossi E, Sabato S.: Psychological factors affecting oncology conditions. Adv Psychosom Med. 2007;28:57-71.

41 Robinson S, Kissane DW, Brooker J, Michael N, Fischer J, Franco M, et al.: Refinement and 
revalidation of the demoralization scale: The DS-II-internal validity. Cancer. 2016 15;122:22512259

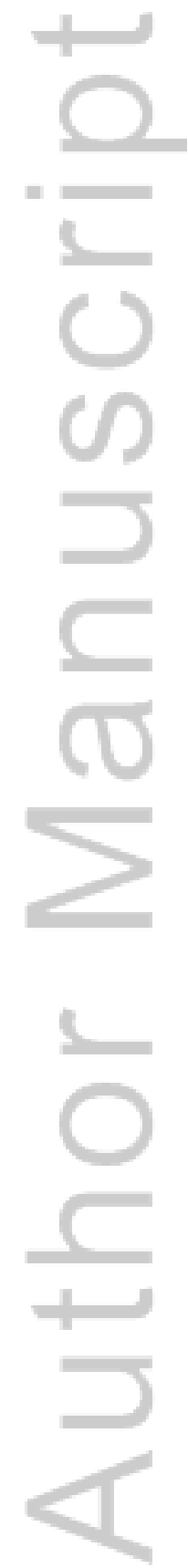

This article is protected by copyright. All rights reserved. 
Table 1. Item and Scale Characteristics (Principal Components Analysis, Varimax Rotated Four-Factor Solution) of the DS-IT

\begin{tabular}{|c|c|c|c|c|c|c|c|c|c|c|}
\hline$(2)$ & \multicolumn{4}{|c|}{ Factor Loadings } & \multicolumn{6}{|c|}{ Items characteristics } \\
\hline Dimensions and Items & F1 & F2 & F3 & $\mathbf{F 4}$ & Mean & SD & $\begin{array}{l}\text { Item Total } \\
\text { correlation }\end{array}$ & $\begin{array}{l}\text { Alpha If } \\
\text { removed }\end{array}$ & Skewness & Kurtosis \\
\hline & & & & & & & & & & \\
\hline \multicolumn{11}{|c|}{ Disheartenment $(8.35 \pm 6.25)$ (explained variance $20.47 \%)$} \\
\hline 21. I feel sad and miserable & 0.74 & & & & 1.03 & 1.05 & .81 & .87 & .62 & -.54 \\
\hline 9. I feel hopeless & 0.73 & & & & .68 & 1.01 & .73 & .88 & 1.37 & .96 \\
\hline 22. I feel discouraged about life & 0.72 & & & & .98 & 1.04 & .72 & .87 & .82 & .03 \\
\hline 18. I feel distressed about what is happening to me & 0.71 & & & & 1.73 & 1.13 & .67 & .88 & .17 & -.78 \\
\hline 24. I feel trapped by what is happening to me & 0.67 & & & & 1.28 & 1.16 & 67 & .88 & .61 & -.43 \\
\hline 5. I no longer feel emotionally in control & 0.63 & & & & 1.09 & 1.09 & .59 & .89 & .65 & -.51 \\
\hline 8. I feel that I cannot help myself & 0.49 & & & 0.42 & .97 & 1.11 & .59 & .89 & .89 & -.03 \\
\hline 23. I feel quite isolated or alone & 0.47 & & & & .57 & 1.01 & .63 & .89 & 1.77 & 2.33 \\
\hline \multirow{2}{*}{\multicolumn{11}{|c|}{ Sense of Failure $(5.86 \pm 3.7)$ (explained variance $12.25 \%)$}} \\
\hline & & & & & & & & & & \\
\hline 19. I am a worthwhile person* & & 0.79 & & & 1.1 & 1.08 & .62 & .73 & 1.07 & .53 \\
\hline 17. I am proud of my accomplishments* & & 0.72 & & & 1.18 & 1.11 & .58 & .74 & .86 & .12 \\
\hline 12. I cope fairly well with life* & & 0.69 & & & 1.18 & 1.04 & .61 & .73 & .93 & .58 \\
\hline 1. There is a lot of value in what I can offer others* & & 0.62 & & & .99 & .89 & .49 & .77 & .91 & 1.1 \\
\hline 6. I am in good spirits* & 0.45 & 0.52 & & & 1.48 & .98 & .53 & .76 & .55 & -.01 \\
\hline 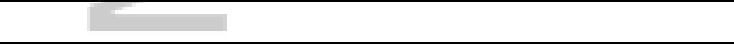 & & & & & & & & & & \\
\hline \multicolumn{11}{|l|}{ Dysphoria $(5.69 \pm 3.88)($ explained variance $12.21 \%)$} \\
\hline 16. I am angry about a lot of things & & & 0.70 & & 1.53 & 1.22 & .59 & .73 & .36 & -.78 \\
\hline 13. I have a lot of regret about my life & & & 0.69 & & 1.02 & 1.13 & .54 & .75 & .85 & -.32 \\
\hline 15. I tend to feel hurt easily & & & 0.67 & & 1.09 & 1.08 & .63 & .72 & .75 & -.13 \\
\hline 11. I feel irritable & & & 0.59 & & 1.46 & 1.17 & .51 & .76 & .41 & -.77 \\
\hline 10. I feel guilty & 0.41 & & 0.42 & & .59 & .98 & .53 & .76 & 1.72 & 2.29 \\
\hline \multicolumn{11}{|c|}{$\begin{array}{l}\text { Loss of meaning and purpose (3.66 } \pm 3.78) \text { (explained variance } \\
12.13 \%)\end{array}$} \\
\hline 7. No one can help me & & & & 0.76 & .93 & 1.27 & .48 & .78 & 1.17 & .17 \\
\hline 14. Life is no longer worth living & & & & 0.64 & .39 & .83 & .56 & .76 & 2.62 & 7.43 \\
\hline 3. I suffer great anxiety about it & & & & 0.60 & .75 & 1.1 & .58 & .75 & 1.51 & 1.57 \\
\hline 4. My role in life has been lost & & & & 0.51 & .58 & .95 & .63 & .74 & 1.61 & 1.85 \\
\hline 2. My life seems to be pointless & 0.45 & & & 0.49 & .71 & .93 & .65 & .74 & 1.14 & .56 \\
\hline 20. I would rather not be alive & & & & 0.46 & .31 & .74 & .41 & .79 & 3.16 & 11.33 \\
\hline
\end{tabular}

This article is protected by copyright. All rights reserved. 
Table 2. Descriptive statistics for DS-IT and other variables and correlations between the measures (Spearman rho)

\begin{tabular}{|l|c|c|c|c|c|c|c|}
\hline & Disheartenment & Meaninglessness & Dysphoria & Failure & Total & $\begin{array}{c}\text { Mini-MAC- } \\
\text { H }\end{array}$ & PHQ-9 \\
\hline Disheartenment & 1 & & & & & $53^{* *}$ & $.65^{* *}$ \\
\hline Meaninglessness & $.61^{* *}$ & 1 & & & & $41^{* *}$ & $41^{* *}$ \\
\hline Dysphoria & $.57^{* *}$ & $.41^{* *}$ & 1 & & & $.33^{* *}$ & $.45^{* *}$ \\
\hline Failure & $.46^{* *}$ & $.47^{* *}$ & $.43^{* *}$ & 1 & & $.46^{* *}$ & $.41^{* *}$ \\
\hline Total & $.87^{* *}$ & $.77^{* *}$ & $.74^{* *}$ & $.72^{* *}$ & 1 & $.54^{* *}$ & $.62^{* *}$ \\
\hline & & & & & & &. \\
\hline Karnofsky score & -.05 & -.08 & .11 & .01 & &. .12 &. .01 \\
\hline $\begin{array}{l}\text { Time since } \\
\text { diagnosis }\end{array}$ & $.19^{*}$ & 0.7 & $.22^{* *}$ & $.17^{*}$ & $.22^{* *}$ & .14 & .09 \\
\hline Age & .01 & $.17^{*}$ & -.11 & .003 & -.02 & .03 & -.01 \\
\hline Mean \pm SD & $8.33 \pm 6.25$ & $3.66 \pm 3.78$ & $5.69 \pm 3.88$ & $5.86 \pm 3.71$ & $23.55 \pm 14.01$ & $10.21 \pm 3.48$ & $5.67 \pm 4.21$ \\
\hline
\end{tabular}

PHQ-9= Patient Health Questionnaire-9; , DS-IT= Demoralization Italian scale; Mini-MAC H= Hopelessness

$* \mathrm{p}<0.05 ; * * \mathrm{p}<0.01$

This article is protected by copyright. All rights reserved. 
Table 3. Cross-tabulation frequencies (n) between the categories of demoralization and the presence of depression (PHQ-9).

\begin{tabular}{|c|c|c|c|c|}
\hline \multirow[t]{2}{*}{ PHQ-9 case vs non-case } & \multicolumn{4}{|c|}{$\begin{array}{c}\text { DS Category } \\
\end{array}$} \\
\hline & No/Low (43) & $\begin{array}{c}\text { Low/Moderate } \\
\text { (104) }\end{array}$ & Moderate/High (17) & High (33) \\
\hline \multicolumn{5}{|l|}{ Non-Case $(\leq 9)^{\mathrm{a}}$} \\
\hline$\%$ of total & $21.6 \%$ & $43.8 \%$ & $5.7 \%$ & $8.2 \%$ \\
\hline Count & $42(97.7 \%)$ & $85(81.7 \%)$ & $11(78.6 \%)$ & $16(48.5 \%)$ \\
\hline Expected count & 34.1 & 82.6 & 11.1 & 26.2 \\
\hline 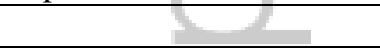 & & & & \\
\hline \multicolumn{5}{|l|}{ Case $(\geq 10)^{\mathrm{a}}$} \\
\hline$\%$ of total & $0.5 \%$ & $9.8 \%$ & $1.5 \%$ & $8.8 \%$ \\
\hline Count & $1(2.3 \%)$ & $19(18.3 \%)$ & $3(21.4 \%)$ & $17(42.5 \%)$ \\
\hline Expected count & 8.9 & 21.4 & 2.9 & 6.8 \\
\hline
\end{tabular}

$\chi^{2} 28.5$, df $3, p=0.0001$.

This article is protected by copyright. All rights reserved. 


\section{University Library}

\section{- M M N E R VA A gateway to Melbourne's research publications}

Minerva Access is the Institutional Repository of The University of Melbourne

Author/s:

Grassi, L;Costantini, A;Kissane, D;Brunetti, S;Caruso, R;Piazza, G;Marchetti, P;Sabato, S;Nanni, MG

Title:

The factor structure and use of the Demoralization Scale (DS-IT) in Italian cancer patients

Date:

2017-11-01

Citation:

Grassi, L., Costantini, A., Kissane, D., Brunetti, S., Caruso, R., Piazza, G., Marchetti, P., Sabato, S. \& Nanni, M. G. (2017). The factor structure and use of the Demoralization Scale (DS-IT) in Italian cancer patients. PSYCHO-ONCOLOGY, 26 (11), pp.1965-1971. https:// doi.org/10.1002/pon.4413.

Persistent Link:

http://hdl.handle.net/11343/292708 